\title{
Money in Gas-Like Markets: Gibbs and Pareto Laws
}

\author{
Arnab Chatterje日 and Bikas K. Chakrabarti \\ Saha Institute of Nuclear Physics, Block-AF, Sector-I Bidhannagar, Kolkata-700064, India. \\ S. S. Manna \\ Satyendra Nath Bose National Centre for Basic Sciences Block-JD, Sector-III, Salt Lake, Kolkata-700098, India.
}

\begin{abstract}
We consider the ideal-gas models of trading markets, where each agent is identified with a gas molecule and each trading as an elastic or money-conserving (two-body) collision. Unlike in the ideal gas, we introduce saving propensity $\lambda$ of agents, such that each agent saves a fraction $\lambda$ of its money and trades with the rest. We show the steady-state money or wealth distribution in a market is Gibbs-like for $\lambda=0$, has got a non-vanishing most-probable value for $\lambda \neq 0$ and Pareto-like when $\lambda$ is widely distributed among the agents. We compare these results with observations on wealth distributions of various countries.
\end{abstract}

PACS numbers: 87.23.Ge;89.90.+n;02.50.-r

\section{INTRODUCTION}

In an earlier conference in Kolkata in 1994, many leading Indian economists from the Indian Statistical Institute and physicists met and discussed about the possible formulations of some economic problems and their solutions using tricks from physics [1]. In one of these papers 2], possibly the first published joint paper with both physicist and economist Indian coauthors, the possibility of a kinetic theory of (ideal) gas-like model of trading in the market was discussed. Among other things, it tried to identify, from the known effects of various fiscal policies, equivalence of the (kinetic) energy of the gas molecules (money) and the temperature (average money in the market). Such a "finite temperature" market model and the corresponding distributions were also noted by others [3, 4]. With the possibility of putting more than one agent in the same (micro) state, identified by the price or money income of the agent in the market, the likely distribution was concluded there to be Bose-Einstein like, rather than Gibbs like [2]. These studies of course had the limitation of absence of any comparison with real income distributions (in any market or country). In a recent paper by Dragulescu and Yakovenko [4] a simple (trading) market model was developed with fixed (total) money and number of agents in the market. Random two-agent exchanges (with local money conservation) lead to Gibbslike steady income distribution. This was also confirmed by simple numerical simulations. Modifications due to savings was studied simultaneously 5 . In a very recent review [6], a popular introduction to these developments is given.

Saving propensity among the agents, a very selfish and local feature of the tradings, introduce in effect some global co-operative feature (cf. [5]). We show that a fixed and uniform saving propensity of all the agents in

*Electronic address: arnab@cmp.saha.ernet.in the market shifts the most-probable money of the distribution away from zero (as given by Gibbs for zero savings), while a random distribution of saving propensity among the population can give the Pareto (power) law 7]

$$
P(m) \sim m^{-(1+\nu)}
$$

for the wealth or money $(m)$ distribution. We intend to discuss here in brief the effects of various kinds of savings on the ideal gas-like money distributions in the abovementioned market models, and compare our observations with those from real markets.

\section{AN IDEAL GAS-LIKE MARKET MODEL}

Let us consider a simple model of a closed economic system where the total amount of money $M$ and the total number $N$ of agents are fixed. No development (production) or migration (death/birth of agents) occurs and the only economic activity is confined to trading. Each agent $i$, individual or a corporate, possess a money $m_{i}(t)$ at (discretised) time $t$. Time changes after each trading. In any trading, two randomly chosen agents $i$ and $j$ exchange their money such that their total money is (locally) conserved and none ends up with negative money (debt not allowed):

$$
m_{i}(t)+m_{j}(t)=m_{i}(t+1)+m_{j}(t+1)
$$

where $m_{i}(t) \geq 0$ for all $i$ and $t ; \sum_{i=1}^{N} m_{i}=M$. Since money is conserved, in the steady state $(t \rightarrow \infty)$, the probability $P(m)$ of the density of people with money $m$ will satisfy 
which corresponds to the Gibbs distribution [4, 5]

$$
P(m)=(1 / T) \exp (-m / T) ; \quad T=M / N .
$$

Numerical simulations in the model also confirmed the steady state distribution of money, no matter what initial distribution of money the agents had, to be Gibbs' one: after sufficient number of tradings, most of the agents end up with very little money! This result is quite robust (and realistic too!). In fact, several variations of the trading, and of the 'lattice' (on which the agents can be put and each agent trade with its 'lattice neighbours' only), whether compact, fractal or small-world [3], leaves the distribution unchanged. Some other variations like random sharing of an amount $2 m_{2}$ only (not of $m_{1}+m_{2}$ ) when $m_{1}>m_{2}$ (trading at the level of lower economic class), lead to even drastic situation: all the money in the market drifts to one agent and the rest of the agents all become truely pauper [6, 8] ! Attempts have also been made [9] to get Pareto-like power-law distribution here with changed definition of entropy or the conservation law (cf. eqn. (3)).

Chakrabarti and Marjit 2] argued for the BoseEinstein like distribution (rather than Gibbs) in such a market (with the temperature $T$ similarly identified with the average money $M / N$ per agent), as one can put more than one agent in the same economic state (specified by the income) and the maximisation of the consequent entropy. For the possibility of adding and subtracting agents into/from the market, one similarly needs (negative) "chemical potential" which becomes zero at a finite temperature or money level in the market, when the "Bose condensed" fraction of the agents will fall out of the market distribution and might be identified as unemployed.

The real income distributions did not indicate so far anything like the Bose distribution; rather considerable evidences support the possibility of Gibbs like distribution (4) in the income (almost for $90 \%$ of the low-income range) of various countries (see e.g., [10], see also data in 11]; Fig. 2).

\section{MODEL WITH FIXED SAVING PROPENSITY OF THE AGENTS}

Here we assume [5] that each economic agent $i$ saves a fraction $\lambda$ of its money $m_{i}(t)$ before the trading at time $t$. We again assume that an agent's money is nonnegative and no debt is permitted. Let us now consider an arbitrary pair of agents $i$ and $j$, who get engaged in a trade, and their money $m_{i}(t)$ and $m_{j}(t)$ before the trade change respectively to

$$
m_{i}(t+1)=m_{i}(t)+\Delta m ; \quad m_{j}(t+1)=m_{j}(t)-\Delta m
$$

$$
\text { where } \quad \Delta m=(1-\lambda)\left[\epsilon\left\{m_{i}(t)+m_{j}(t)\right\}-m_{i}(t)\right] \text {; }
$$

with $\epsilon$ as any random fraction. As may be checked by straight-forward substitution, this kind of trading again satisfies eqn. (2), while each agent saves a fixed fraction $\lambda$ of its money before the trade and exchanges randomly (with fraction $\epsilon$ ) the rest of the money.

One finds here that at $\lambda=0$ the market becomes noninteracting and the steady state money distribution becomes the Gibbs' one. For any nonvanishing $\lambda$, the equilibrium distribution becomes asymmetric Gibbs-like (see inset of Fig. 1) with the most-probable money $m_{p}$ per agent (corresponding to the peak in $P(m)$ ) shifting away from $m=0$ (for $\lambda=0$ ) to $M / N$ as $\lambda \rightarrow 1$ [5]. This self-organising feature of the market, induced by sheer self-interest of saving by each agent without any global perspective, is very significant as the fraction of paupers decreases with saving fraction $\lambda$ and most people end up with the average money in the market (socialists' dream achieved with just people's self-interest of saving)! Interestingly, self-organisation also occur in such market models when there is restriction in the commodity market 12. Although this fixed saving propensity does not give yet the Pareto-like power-law distribution, the Markovian nature of the scattering or trading processes (eqn. (31) is lost and the system becomes co-operative. Indirectly through $\lambda$, the agents get to know (start interacting with) each other and the system co-operatively selforganise towards a most probable distribution $\left(m_{p} \neq 0\right)$.

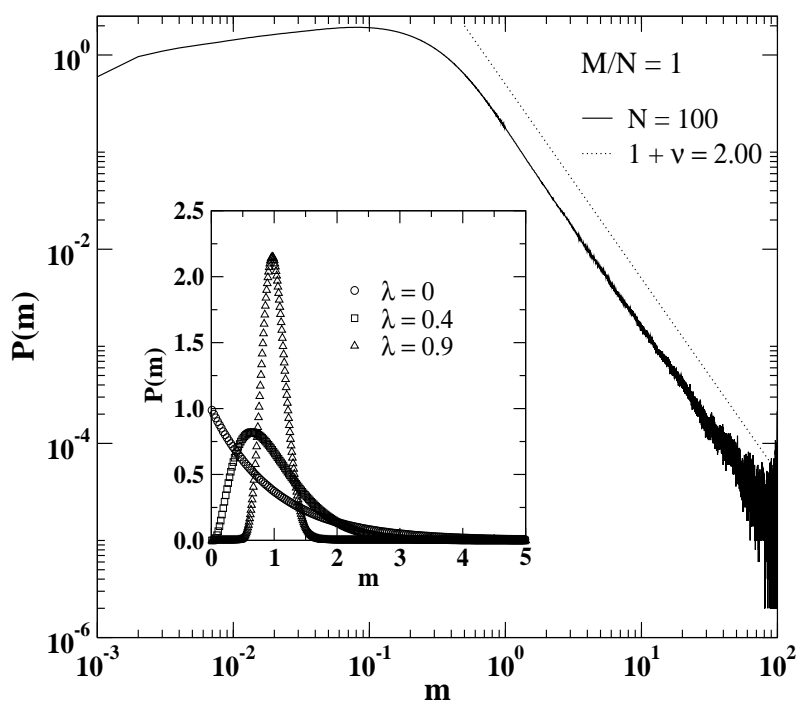

FIG.1: Money distribution $P(m)$ in the model for distributed $\lambda$ $(0 \leq \lambda<1)$. Inset shows $P(m)$ for three typical values in the fixed $\lambda$ case (including $\lambda=0$; Gibbs). For both cases, $N=100$ play with average money per agent $M / N=1$.

\section{MODEL WITH RANDOM SAVING PROPENSITY OF THE AGENTS}

We now consider a market again with fixed $N$ and $M$ but with random saving propensity $\lambda_{i}\left(0 \leq \lambda_{i}<1\right)$ fixed or "quenched" for each agent $\left(\lambda_{i}\right.$ are independent of 
trading or $t$, but vary randomly from agent to agent) [13]. One again follows the same trading rules as mentioned in the previous section (eqn. (5)), except that

$$
\Delta m=\epsilon\left(1-\lambda_{j}\right) m_{j}(t)-\left(1-\lambda_{i}\right)(1-\epsilon) m_{i}(t)
$$

here; $\lambda_{i}$ and $\lambda_{j}$ are the saving propensities of agents $i$ and $j$. We first take a market with $N$ agents, each having a fixed saving propensity $\lambda$ distributed independently, randomly and uniformly (white) within an interval 0 to 1 . Having assigned each agent $i$ the saving propensities $\lambda_{i}$, and starting with an arbitrary initial (uniform or random) distribution of money among the agents, we start the tradings. At each time, two agents are randomly selected and the money exchange among them occurs, following the above mentioned scheme. We check for the steady state, by looking at the stability of the money distribution $P(m)$ in successive Monte Carlo steps $t$.

In Fig. 1, we show the money distribution $P(m)$ vs. $m$ (in units of $M / N$ ) for $N=100, M / N=1$, after averaging over $10^{6}$ initial configurations $\left(\lambda_{i}\right.$ distribution among the agents) at $t / N=10,000$. There is an initial growth of $P(m)$ from $m=0$, which quickly saturates and then a long range of power-law decay in $P(m)$ for large $m$ values (for less than $10 \%$ of the population $N$ in the market) is observed (for more than two decades in $m$ ). This decay, when fitted to Pareto law (1), gives $\nu=1.03 \pm 0.03$.



FIG.2: Cumulative distribution $Q(m)=\int_{m}^{\infty} P(m) d m$ of wealth $m$ in USA [10] in 1997 and Japan [11] in 2000. Low-income group follow Gibbs law (shaded region) and the rest (about 5\%) of the rich population follow Pareto law. The inset shows the cumulative distribution for a model market where $p=0.9$ fraction of the agents trade randomly without any saving and the rest $1-p$ fraction trades with their saving propensities distributed uniformly between 0 and 1. The dotted line (for large $m$ values) corresponds to $\nu=1.0$.

We now investigate on the range of distribution of the saving propensities within the population. If a certain fraction $p$ of the population trades in the market randomly without any saving while the rest $(1-p)$ fraction have a quenched distribution of their saving propensities $\left(0 \leq \lambda_{i}<1\right)$, we observe that for larger values of $p$ $(p>0.8)$, the distribution is Gibbs-like for low-income group and has a power law tail for the high-income group. The range of validity of Gibbs law increases with increasing $p$. However, the exponent $\nu$ does not change with $p$. These model wealth distributions $P(m)$ compare very well with the wealth distributions of various countries: Data suggests Gibbs like distribution in the low-income range [10] (more than 90\% of the population) and Paretolike in the high-income range [1] (less than 10\% of the population) of various countries (Fig. 2).

We also considered annealed randomness an the saving propensity $\lambda$ : here $\lambda_{i}$ for any agent $i$ changes from one value to another within the range $0 \leq \lambda_{i}<1$, after each trading. Numerical studies for this annealed model did not show any power law behavior for $P(m)$; rather it again becomes exponentially decaying on both sides of a most-probable value $m_{p}(\lambda)$, similar to fixed $\lambda$ case.

\section{SUMMARY AND CONCLUSIONS}

We have considered ideal-gas models of trading markets. In these models, we introduce saving propensity $\lambda$ of agents, such that each agent saves a fraction $\lambda$ of its money and trades with the rest. We show the steadystate money or wealth distribution $P(m)$ in the market is that of Gibbs (4) for $\lambda=0$, has got a non-vanishing most-probable value for $\lambda>0$ (but fixed for all agents), and one gets Pareto distribution (11) with $\nu \simeq 1.0$ when $\lambda$ is widely distributed among the agents. These results in simple ideal-gas like market models also compare well with real market observations.
[1] Proc. School on Complex Systems, (1995) Indian J. Phys. B 69483

[2] B. K. Chakrabarti, S. Marjit (1995), Indian J. Phys. B 69681.

[3] S. Moss de Oliveira, P. M. C. de Oliveira, D. Stauffer
(1999), Evolution, Money, War and Computers, Tuebner, Stuttgart.

[4] A. A. Dragulescu, V. M. Yakovenko (2000), Euro. Phys. J. B 17723.

[5] A. Chakraborti, B. K. Chakrabarti (2000), Euro. Phys. 
J. B 17167.

[6] B. Hayes (2002), Am. Scientist, 90400.

[7] V. Pareto (1897), Le Cours d'Economique Politique, Lausanne \& Paris.

[8] A. Chakraborti (2002), arXiv:cond-mat/0205221, Int. J. Mod. Phys. C 131315.

[9] C. Tsallis (2003), An Unifying Concept for Discussing Statistical Physics and Economics, H. Reiss and P. K. Rawlings (2003), The Natural Role of Entropy in Equilibrium Economics, in Application of Econophysics: Proc. 2nd Nikkei Econophys. Symp. (Tokyo, 2002), ed. H. Takayasu, (Springer, 2003), in press;

[10] A. A. Dragulescu, V. M. Yakovenko (2001), Euro. Phys. J. B 20 585; A. A. Dragulescu, V. M. Yakovenko (2002),
arXiv:cond-mat/0211175

[11] Y. Fujiwara, H. Aoyama (2003), in Application of Econophysics: Proc. 2nd Nikkei Econophys. Symp. (Tokyo, 2002), ed. H. Takayasu, (Springer, 2003), in press; arXiv:cond-mat/0208398

[12] A. Chakraborti, S. Pradhan, B. K. Chakrabarti (2001), Physica A 297253.

[13] A. Chatterjee (2002) unpublished; A. Chatterjee, B. K. Chakrabarti, S. S. Manna (2003), arXiv: cond-mat/0301289 B. K. Chakrabarti and A. Chatterjee in Application of Econophysics: Proc. 2nd Nikkei Econophys. Symp. (Tokyo, 2002), ed. H. Takayasu, (Springer, 2003), in press; cond-mat/0302147 